\title{
石墨烯量子点/铁基金属-有机骨架复合材料高效光催化二氧化碳还原*
}

\author{
王旭生 $b, c, g$ 杨胥 $a, b$ 陈春辉 $b, d$ 李红芳 $a, b$ \\ 黄远标*,a,b,f 曹荣 $a, b, e, f$

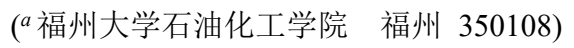 \\ ( ${ }^{b}$ 中国科学院福建物质结构研究所结构化学国家重点实验室 福州 350002) \\ (c 浙江理工大学材料科学与工程学院 杭州 310018) \\ ( ${ }^{d}$ 中国科学技术大学化学系 合肥 230026) \\ ( $e$ 中国福建光电信息科学与技术创新实验室(闽都创新实验室) 福州 350108) \\ ( $f$ 中国科学院大学 北京 100049) \\ ( $g$ 暨南大学化学与材料学院 广州 510632)
}

\begin{abstract}
摘要 利用可见光将二氧化碳光还原为有用的化学品是一项有前景但充满挑战的工作. 金属有机骨架(MOFs)作为一种 新兴的具高孔隙率、高比表面积、强吸附富集 $\mathrm{CO}_{2}$ 能力、结构和功能可调的多孔材料, 在光催化二氧化碳还原反应中 具有极强的应用潜力. 但大多数金属有机骨架材料存在可见光吸收范围窄、光生载流子快速复合等问题，导致催化二 氧化碳还原活性仍然较低. 通过静电自组装策略将纳米级胺基化金属有机骨架材料 $\left(\mathrm{NH}_{2}-\mathrm{MIL}-88 \mathrm{~B}(\mathrm{Fe})\right.$ )和羧酸化石墨 烯量子点(GQD)通过静电作用结合，得到 GQD/ $\mathrm{NH}_{2}-\mathrm{MIL}-88 \mathrm{~B}(\mathrm{Fe})$ 复合材料. 该复合催化剂有效结合了金属有机骨架强 二氧化碳吸附富集能力和 GQD 的可见光吸收范围宽、电子传导能力强等优点, 因此与纯金属有机骨架材料 $\mathrm{NH}_{2}-$ MIL-88B $(\mathrm{Fe})$ 相比较, 该复合材料能高效光催化还原 $\mathrm{CO}_{2}$ 为 $\mathrm{CO}$, 并在 $10 \mathrm{~h}$ 可见光下活性高达 $590 \mu \mathrm{mol} / \mathrm{g}$, 约为 $\mathrm{NH}_{2}-\mathrm{MIL}-88 \mathrm{~B}(\mathrm{Fe})$ 活性的四倍. 这项工作为制备高活性催化 $\mathrm{CO}_{2}$ 的金属有机骨架复合材料提供了借鉴.
\end{abstract}

关键词 金属有机骨架; 光催化; 二氧化碳还原; 静电自组装; 石墨烯量子点

\section{Graphene Quantum Dots Supported on Fe-based Metal-Organic Frameworks for Efficient Photocatalytic $\mathrm{CO}_{2}$ Reduction ${ }^{*}$}

\author{
Wang, Xusheng ${ }^{b, c, g} \quad$ Yang, $\mathrm{Xu}^{a, b}$ \\ Huang, Yuanbiao*,a,b,f \\ Chen, Chunhui ${ }^{b, d}$ \\ Cao, Rong ${ }^{a, b, e, f}$ \\ Li, Hongfang ${ }^{a, b}$ \\ ( ${ }^{a}$ College of Chemical Engineering, Fuzhou University, Fuzhou 350108) \\ ( ${ }^{b}$ State Key Laboratory of Structural Chemistry, Fujian Institute of Research on the Structure of Matter, \\ Chinese Academy of Sciences, Fuzhou 350002) \\ ( ${ }^{c}$ School of Materials Science and Engineering, Zhejiang Sci-Tech University, Hangzhou 310018) \\ ( ${ }^{d}$ Department of Chemistry, University of Science and Technology of China, Hefei 230026) \\ ( ${ }^{e}$ Fujian Science \& Technology Innovation Laboratory for Optoelectronic Information of China, Fuzhou 350108) \\ (f University of Chinese Academy of Sciences, Beijing 100049) \\ ( ${ }^{g}$ College of Chemistry and Materials Science, Jinan University, Guangzhou 510632)
}

Abstract Photocatalytic reduction of $\mathrm{CO}_{2}$ to valuable chemicals is an essential but still remains challenging. Metal-organic frameworks (MOFs) featuring high special surface area, large $\mathrm{CO}_{2}$ adsorption uptakes, adjustable structures and function, have become a kind of promising porous materials for photocatalytic $\mathrm{CO}_{2}$ reduction. However, MOFs often suffer from problems like short light harvesting range, rapid recombination of photogenerated carriers, resulting in lower activity. Here,

\footnotetext{
* Dedicated to the 10th anniversary of the Youth Innovation Promotion Association, CAS.

* E-mail: ybhuang@fjjirsm.ac.cn

Received October 13, 2021; published November 29, 2021.

Supporting information for this article is available free of charge via the Internet at http://sioc-journal.cn.

Project supported by the National Key Research and Development Program of China (Nos. 2018YFA0208600, 2018YFA0704502), the National Natural Science Foundation of China (Nos. 21671188, 21871263, 22071245, 22033008, 22171265 and 22001094), the Youth Innovation Promotion Association, CAS (Y201850), Fujian Science \& Technology Innovation Laboratory for Optoelectronic Information of China (No. 2021ZZ103), and Guangdong Basic and Applied Basic Research Foundation (No. 2020A1515110003).

*庆祝中国科学院青年创新促进会十年华诞.

项目受国家重点基础研发计划项目(Nos. 2018YFA0208600, 2018YFA0704502)、国家自然科学基金(Nos. 21671188, 21871263, 22071245, 22033008, $22171265,22001094) 、 中$ 国科学院青年创新促进会优秀会员项目(No. Y201850)、中国福建光电信息科学与技术创新实验室(闽都创新实验室)项目(No. 2021ZZ103)和广东省基础与应用基础研究基金(No. 2020A1515110003)资助.
} 
graphene quantum dots (GQD) were supported on the Fe-based nano-sized MOFs, $\mathrm{NH}_{2}-\mathrm{MIL}-88 \mathrm{~B}(\mathrm{Fe})$, via electrostatic self-assembly strategy. GQDs were prepared by electrolysis of graphite rod in pure water firstly, and then centrifuged to remove the large species. Transmission electron microscope (TEM) reveals that ultrafine GQDs with $3 \mathrm{~nm}$ were obtained. Atomic force microscope (AFM) further demonstrates that the thickness of GQDs is around $0.34-1.5 \mathrm{~nm}(1-4$ stacked layers). The MOFs, $\mathrm{NH}_{2}-\mathrm{MIL}-88 \mathrm{~B}(\mathrm{Fe})$, were synthesized with traditional solvothermal method, with a nano spindle shape of $250 \mathrm{~nm} \times 40 \mathrm{~nm}$. The amino groups on MOFs provide strong electrostatic force with the carboxylic groups on GQDs, making the composite very stable and efficient electron transfer. High resolution transmission electron microscope (TEM) reveals that the nano MOFs were surrounded by tiny GQDs firmly. The bandgap of composite was determined by solid ultraviolet visible diffuse reflectance spectroscopy (UV-Vis DRS) and Mott-Schottky measurement, which indicate that it is thermodynamically appropriate for photocatalytic $\mathrm{CO}_{2}$ reduction. Photocurrent experiments further demonstrate the composite is beneficial for the photogenerated electron-hole separation. Thus, the resulting GQD/ $\mathrm{NH}_{2}-\mathrm{MIL}-88 \mathrm{~B}(\mathrm{Fe})$ composite showed much enhanced $\mathrm{CO}$ production rate (4 times) compared with the parent $\mathrm{NH}_{2}-\mathrm{MIL}-88 \mathrm{~B}(\mathrm{Fe})$, reaching $590 \mu \mathrm{mol} / \mathrm{g}$ under $10 \mathrm{~h}$ visible light irradiation with triethanolamine (TEOA) as sacrificial agent. The hugely improved photoreduction activity benefits from both the high $\mathrm{CO}_{2}$ adsorption of MOFs and the enhanced separation of photogenerated electrons and holes. This work provides an avenue for preparation of MOFs based materials with high $\mathrm{CO}_{2}$ photoreduction activity.

Keywords metal-organic frameworks; photocatalysis; $\mathrm{CO}_{2}$ reduction; electrostatic self-assembly; graphene quantum dots

\section{1 引言}

近年来，由于工业上化石燃料的广泛使用，产生了 大量的温室气体如 $\mathrm{CO}_{2}$, 导致全球气温升高、海平面上 升以及生态失衡等问题 ${ }^{[1-4]}$. 如何将 $\mathrm{CO}_{2}$ 捕获利用, 进而 转化为附加值高的化学品是人们一直思考的问题. 利用 太阳光中范围最广的可见光将二氧化碳光还原为有用 的化学品是一项有前景但充满挑战的工作 ${ }^{[5-7]}$. 目前人 们采用多种分子或者半导体催化剂用于光催化二氧化 碳还原, 但是效率仍然较低 ${ }^{[8-11]}$. 这主要是因为较低的 二氧化碳吸附、可见光吸收范围窄、快速的光生载流子 复合等因素造成. 因此, 设计合成高效的新型光催化剂 在可见光下催化还原二氧化碳至关重要.

由无机金属离子或簇与有机配体通过配位键组装 形成的金属有机骨架材料, 是一类新兴的具有高孔隙 率、高比表面积、结构和功能可调的多孔材料, 已经广 泛应用于气体吸附与分离、载药、催化等多种领域 ${ }^{[12-21]}$. 尤其是其具有强的吸附富集 $\mathrm{CO}_{2}$ 的能力能够有效促进 $\mathrm{CO}_{2}$ 的转化 ${ }^{[22-23]}$. 此外, 可以通过直接法或者后修饰方 法调控活性中心、光的吸收范围、能带范围等来促进 $\mathrm{CO}_{2}$ 的催化转化. 鉴于以上优点, 李朝晖等 ${ }^{[24]}$ 将 $\mathrm{NH}_{2}$-MIL-125(Ti) 应用于光催化二氧化碳还原反应, 并 成功地将 $\mathrm{CO}_{2}$ 还原为甲酸. 此后, 一些其它金属有机骨 架材料也相继发现具有光催化二氧化碳还原活性 ${ }^{[25-30]}$. 但是, 纯金属有机骨架材料在光催化 $\mathrm{CO}_{2}$ 还原方面, 仍 然存在可见光吸收范围窄、光生载流子快速复合等问题, 致使其活性仍然较低. 为改善纯金属有机骨架材料光催 化活性，人们将铂、金、银、 $\mathrm{C}_{3} \mathrm{~N}_{4}$ 、石墨烯、 $\mathrm{Zn}_{2} \mathrm{GeO}_{4}$ 等其它组分与金属有机骨架进行复合可以有效改善光 生载流子快速复合的问题, 提升光催化二氧化碳活性, 但仍然难以满足商业化要求 ${ }^{[31-35]}$.

近年来, 石墨烯量子点(GQD) 因其优良的光稳定 性、生物相容性、水溶性以及低毒性, 成为碳纳米材料 中舟苒升起的一颗新星 ${ }^{[36-39]}$. GQD 的直径一般在 $10 \mathrm{~nm}$ 以下, 主要是由 $\mathrm{sp}^{3}$ 杂化的无定形碳和 $\mathrm{sp}^{2}$ 杂化的石墨碳
的纳米晶组成. 尤其是其能够吸收从紫外到近红外的 光, 而且具有良好的发光和电子传导能力, 同时可以作 为电子媒介, 使得它们成为潜在的光催化助催化 剂[40-41].

为解决当前纯金属有机骨架光催化还原 $\mathrm{CO}_{2}$ 活性 低的问题, 本工作拟通过静电自组装策略将胺基化金属 有机骨架材料 $\left(\mathrm{NH}_{2}-\mathrm{MIL}-88 \mathrm{~B}(\mathrm{Fe})\right)$ 和羧酸化石墨烯量子 点 $(\mathrm{GQD})$ 结合, 组装得到 GQD/ $\mathrm{NH}_{2}-\mathrm{MIL}-88 \mathrm{~B}(\mathrm{Fe})$ 复合材 料. 该复合催化剂可以结合金属有机骨架和石墨烯量子 点的优点, 克服纯金属有机骨架材料催化活性低的缺 点, 用于高效光催化 $\mathrm{CO}_{2}$ 还原.

\section{2 结果与讨论}

参考文献方法, 通过电解石墨棒制备了石墨烯量子 点 ${ }^{[22-43]}$. 在烧杯中放入去离子水, 并将两个高纯石墨棒 置入其中, 作为阴极和阳极两个电极. 烧杯用保鲜膜盖 好, 防止电解过程中水的蒸发和灰尘的落入. 保鲜膜上 扎一些小洞, 保证电解出的氢气和氧气可以顺利排出. 在 $30 \mathrm{~V}$ 直流电源的电压作用下，阳极石墨电极逐渐分 解，15 d 左右以后，阳极石墨棒电极在水中部分基本分 解完全, 水的颜色逐渐从无色变成淡黄色, 最后变成黑 色, 说明产生了碳点. 电解后的电解液先经滤纸过滤, 去除大颗粒石墨, 随后经 $20000 \mathrm{r} / \mathrm{min}$ 的高速离心机离 心, 去除剩余颗粒较大的石墨和石墨烯, 得到包含 GQD 的上清液. 如图 1a 所示, GQD 水溶液的颜色是淡棕色. 紫外可见吸收光谱显示, 合成的 GQD 水溶液在 $229 \mathrm{~nm}$ 有一个很强的吸收峰(图 S1, 支持信息), 可以归属于 $\pi-\pi *$ 的电子跃迁 ${ }^{44]}$. 另外, 在 $365 \mathrm{~nm}$ 紫外灯照射下, 合 成的 GQD 水溶液有蓝色荧光发射现象(图 1b). 进一步 的激发波长依赖的苂光发射光谱显示(图 1c), 合成的 GQD 在不同波长光源激发下，展现出不同的荧光发射 谱, 说明合成的 GQD 具有激发波长依赖的苂光发射特 点. 激发波长依赖的发射行为可能是因为同一个 GQD 具有多种荧光发射位点, 或不同 GQD 具有不同的荧光 
发射位点造成的. 这种现象和以前报道的结果类似 ${ }^{[42]}$.

在石墨烯拉曼光谱中, D 峰通常被认为是石墨的缺 陷或边界峰，一般位于 $1270 \sim 1450 \mathrm{~cm}^{-1}$. G 峰代表石墨 烯中 $\mathrm{sp}^{2}$ 杂化碳原子的特征峰, 一般位于 $1587 \mathrm{~cm}^{-1}$ 处. 2D 峰代表双声子共振拉曼峰, 反映的是石墨烯材料的 堆叠层数和方式. 氧化石墨(电解液滤渣)的拉曼光谱 $\left(\lambda_{\mathrm{ex}}\right.$ $=633 \mathrm{~nm}$ )显示(图 1d), 在 $1000 \sim 3000 \mathrm{~cm}^{-1}$ 范围有 1340 , $1587,2670 \mathrm{~cm}^{-1}$ 三个特征峰, 分别对应 D、G 和 $2 \mathrm{D}$ 峰. 其中 $I_{\mathrm{D}} / I_{\mathrm{G}} \approx 1$, 表明石墨电极在被电解后, 出现了大量 的缺陷. $I_{2 \mathrm{D}} / I_{\mathrm{G}}<<1$, 且 $2 \mathrm{D}$ 峰的半峰宽约为 $90 \mathrm{~cm}^{-1}$, 表 明氧化石墨的层数较多. 经滤纸过滤和高速离心, 去除 大颗粒后得到的 GQD 的拉曼光谱在 $1000 \sim 3000 \mathrm{~cm}^{-1}$ 范围仅有两个特征峰, 分别为 $1342 \mathrm{~cm}^{-1}$ 处的 D 峰和 $1608 \mathrm{~cm}^{-1}$ 处的 $\mathrm{G}$ 峰, 同时, $I_{\mathrm{D}} / I_{\mathrm{G}}$ 与氧化石墨(电解液滤 渣)类似, 表明 GQD 同样具有较高的缺陷. 相对氧化石 墨(电解液滤渣), GQD 的 2D 峰基本消失, 说明石墨烯量 子点的形成 ${ }^{[42]}$.

阳极石墨电极被直流电压持续作用下，逐渐氧化分 解. 因此, 我们猜测通过电解法得到的 GQD 具有大量 的亲水性 $\mathrm{O}-\mathrm{C}=\mathrm{O}$ 和 $\mathrm{C}-\mathrm{O}-\mathrm{C}$ 官能团. 在 $\mathrm{GQD}$ 的红 外光谱中(图 1e), $1709 \mathrm{~cm}^{-1}$ 归属为羧基中 $\mathrm{C}=\mathrm{O}$ 伸缩振 动, $3435 \mathrm{~cm}^{-1}$ 归属为 $\mathrm{OH}$ 振动. $1238 \mathrm{~cm}^{-1}$ 归属为 $\mathrm{C}-\mathrm{O}$ $-\mathrm{C}$ 环氧键反对称伸缩振动 ${ }^{[45]}$. 这些官能团证实了我们 合成的 GQD 中确实还有大量的 $\mathrm{O}-\mathrm{C}=\mathrm{O}$ 和 $\mathrm{C}-\mathrm{O}-\mathrm{C}$ 官能团. 另外, GQD 的红外光谱中的 $1448 \mathrm{~cm}^{-1}$ 归属为
芳香性 $\mathrm{C}=\mathrm{C}$ 双键，证明了 $\mathrm{GQD}$ 仍然保持了石墨烯的结 构. 进一步通过 X 射线光电子能谱对 GQD 中的官能团 进行验证. 如图 1f 所示, GQD 的 $X$ 射线光电子高分辨碳 谱显示, $\mathrm{GQD}$ 中含有大量的 $\mathrm{O}-\mathrm{C}=\mathrm{O}$ 和 $\mathrm{C}-\mathrm{O}-\mathrm{C}$ 官能 团, 结合能分别为 288.92 和 $285.55 \mathrm{eV}$, 这和红外数据分 析结果相符. 同时, $284.96 \mathrm{eV}$ 归属为 $\mathrm{C}-\mathrm{C}$ 单键, 进一 步证明合成的 GQD 中含有大量的缺陷位点. 另外, $284.0 \mathrm{eV}$ 归属于 $\mathrm{C}=\mathrm{C}$ 双键, 进一步证实石墨烯结构的 保持. GQD 的 X 射线光电子高分辨氧谱(图 $1 \mathrm{~g}$ ) 也验证了 $\mathrm{O}-\mathrm{C}=\mathrm{O}$ 和 $\mathrm{C}-\mathrm{O}-\mathrm{C}$ 官能团的存在(结合能分别为 531.86 和 $533.15 \mathrm{eV})$.

透射电子显微镜可以很直观地测量 GQD 的形貌和 大小. 如图 $2 \mathrm{a}$ 和 $2 \mathrm{~b}$ 所示, 合成的 GQD 尺寸较为均匀, 颗粒大小约为 $3 \mathrm{~nm}$. 进一步高分辨透射电子显微镜显 示(图 2b 插图), 得到的 GQD 保持很高的结晶度, 晶格 间距为 $0.207 \mathrm{~nm}$, 对应石墨烯(101) 晶面 ${ }^{[43]}$. 我们也用了 动态光散射的方法对 GQD 的尺寸进行了测量(表 S1, 支 持信息), 平均尺寸约为 $6.8 \mathrm{~nm}$. 由于动态光散射的方法 测定的是纳米颗粒的水合尺寸，所以与透射电镜观测到 的 GQD 尺寸相比，其尺寸更大. 当然也不能完全排除 GQD 在水中的部分团聚导致尺寸增大. 原子力显微镜 是测量 GQD 厚度和层数的有效手段. 如图 $2 \mathrm{c}$ 和 $2 \mathrm{~d}$ 所 示, 制备的 GQD 的厚度约为 $1.5 \mathrm{~nm}$, 对应的层数约为 4 层, 少量 GQD 甚至可以达到单层, 厚度仅约 $0.34 \mathrm{~nm}$.
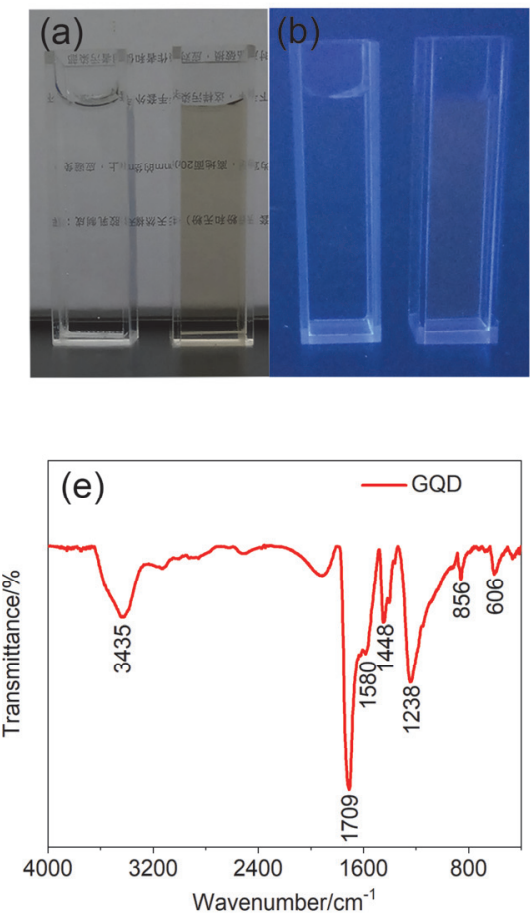
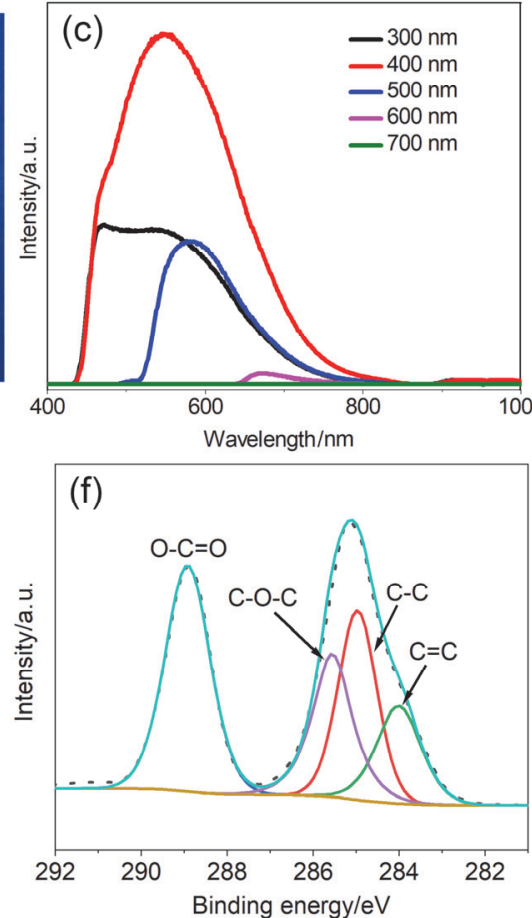
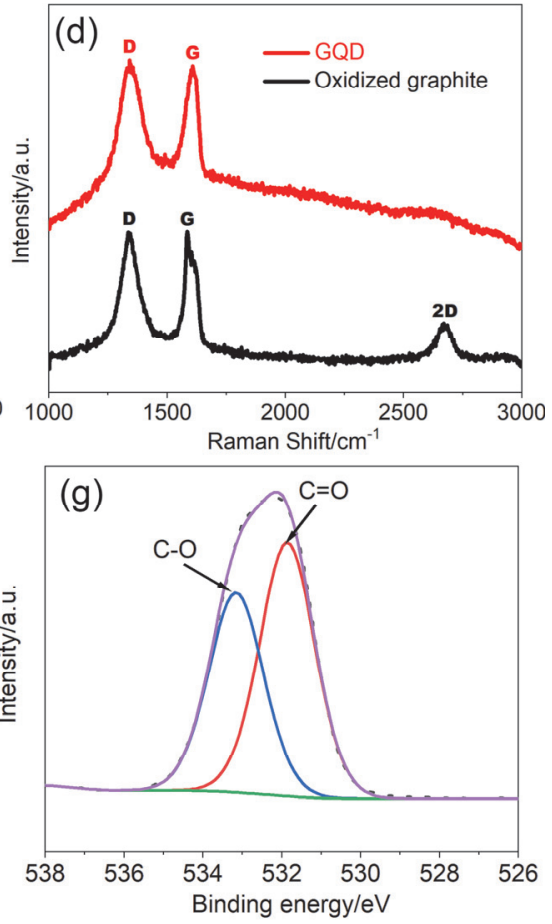

图 1 (a)自然光下蒸馏水(左)和 GQD 水分散液(右)的照片; (b) $365 \mathrm{~nm}$ 紫外光下蒸馏水(左)和 GQD 水分散液(右)的照片; (c) GQD 水分散液在不同 激发波长下的荧光发射谱; (d) GQD 粉末和氧化石墨的拉曼谱图 $\left(\lambda_{\mathrm{ex}}=633 \mathrm{~nm}\right.$ ); (e) GQD 粉末的红外谱图; (f) GQD 粉末的 X 射线光电子高分辨碳谱 $(\mathrm{C} 1 \mathrm{~s}) ;(\mathrm{g}) \mathrm{GQD}$ 粉末的 X 射线光电子高分辨氧谱 $(\mathrm{O} 1 \mathrm{~s})$

Figure 1 (a) The photograph of water (left) and GQD dispersion (right) under sunlight; (b) the photograph of water (left) and GQD dispersion (right) under UV light $(365 \mathrm{~nm})$; (c) the wavelength dependent PL of GQD dispersion; (d) Raman spectra of GQD and oxidized graphite $\left(\lambda_{\mathrm{ex}}=633 \mathrm{~nm}\right)$; (e) IR spectra of GQD powder; (f) C1s XPS spectra of GQD; (g) O1s XPS spectra of GQD 

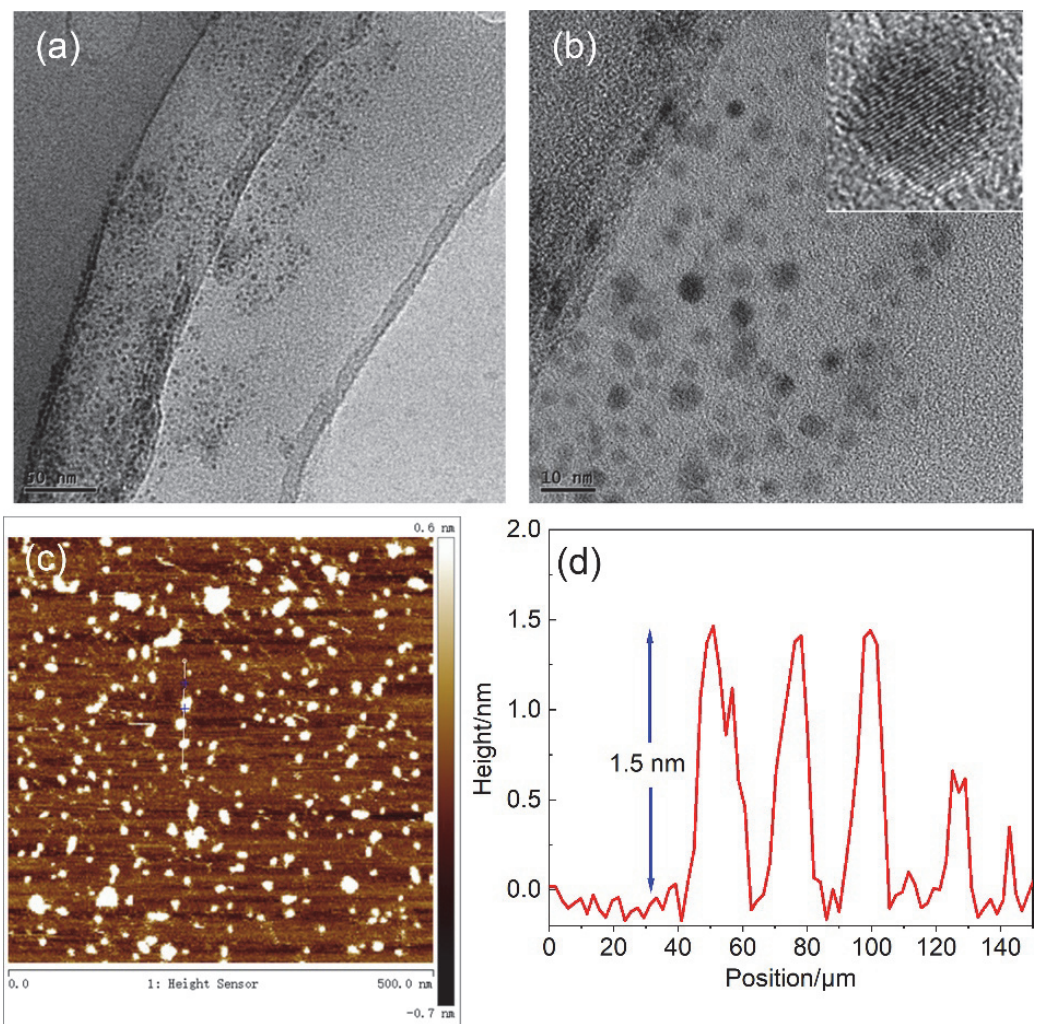

图 2 (a) GQD 的 TEM 图(标尺为 $50 \mathrm{~nm}$ ); (b) GQD 的 TEM 图(标尺为 $10 \mathrm{~nm}$, 插图为 GQD 的高分辨 TEM 图); (c) GQD 的 AFM 图; (d) 所选的 GQD 的高度(c 图中)

Figure 2 (a) The TEM image of GQD (scale bar $50 \mathrm{~nm}$ ), (b) TEM images of GQD (scale bar $10 \mathrm{~nm}$, the inset in b is the HRTEM image of GQD); (c) the AFM image of GQD; (d) the height of selected GQDs in figure (c)

我们随后通过溶剂热法合成了 $\mathrm{NH}_{2}-\mathrm{MIL}-88 \mathrm{~B}(\mathrm{Fe})$. 扫描电子显微镜图像显示(图 $3 \mathrm{a} \sim 3 \mathrm{c}$ ), 合成的 $\mathrm{NH}_{2}$ MIL- $88 \mathrm{~B}(\mathrm{Fe})$ 为尺寸均一的纳米纺锤, 直径约 $40 \mathrm{~nm}$, 长 约 $250 \mathrm{~nm}$. 纳米级尺寸具有更高的表面积, 有利于其后 续与 GQD 混合时的接触面积. 另外, $\mathrm{NH}_{2}-\mathrm{MIL}-$ $88 \mathrm{~B}(\mathrm{Fe})$ 的粉末 $\mathrm{X}$ 射线衍射峰在干湿两种状态下, 均与 MIL- $88 \mathrm{~B}(\mathrm{Fe})$ 的模拟峰一致, 证明 $\mathrm{NH}_{2}-\mathrm{MIL}-88 \mathrm{~B}(\mathrm{Fe})$ 晶 态结构的成功合成 (图 4a). 值得注意的是, 在 $\mathrm{NH}_{2}-\mathrm{MIL}-88 \mathrm{~B}(\mathrm{Fe})$ 孔道中存在溶剂的时候, 孔径较大, 在较小 $2 \theta$ 角度有衍射峰. 当去除 $\mathrm{NH}_{2}-\mathrm{MIL}-88 \mathrm{~B}(\mathrm{Fe})$ 孔道 中溶剂后, 孔道收缩, 并伴随 $2 \theta$ 角右移. 这种现象证明 了 $\mathrm{NH}_{2}-\mathrm{MIL}-88 \mathrm{~B}(\mathrm{Fe})$ 材料具有柔性的框架结构.

如前文所示, 所制备的 GQD 表面存在大量的羧基 官能团. 而 $\mathrm{NH}_{2}-\mathrm{MIL}-88 \mathrm{~B}(\mathrm{Fe})$ 的表面存在大量的胺基官 能团. 这两种酸碱性质相反的官能团为 GQD 和 $\mathrm{NH}_{2}-\mathrm{MIL}-88 \mathrm{~B}(\mathrm{Fe})$ 的紧密结合提供了可能性. 我们将一 定量的 GQD 和 $\mathrm{NH}_{2}-\mathrm{MIL}-88 \mathrm{~B}(\mathrm{Fe})$ 在水中物理混合均匀 后, 经离心和洗涤, 并真空干燥后得到 $\mathrm{GQD} / \mathrm{NH}_{2}-$ MIL- $88 \mathrm{~B}(\mathrm{Fe})$, 其中 GQD 的质量分数为 $2 \%$. 高分辨透 射电子显微镜表明, GQD 紧密包裹在 $\mathrm{NH}_{2}-\mathrm{MIL}-88 \mathrm{~B}(\mathrm{Fe})$ 的周围(图 3d 3f), 同时, 负载后的 GQD 的晶格间距和 负载前相同(图 3f 插图).
固体紫外可见漫反射光谱显示, $\mathrm{NH}_{2}-\mathrm{MIL}-88 \mathrm{~B}(\mathrm{Fe})$ 的吸光范围可达 $750 \mathrm{~nm}$ (图 4b), 进而计算出其带宽为 $1.9 \mathrm{eV}$ (图 4c). 穆特-肖特基曲线测量出 $\mathrm{NH}_{2}$-MIL$88 \mathrm{~B}(\mathrm{Fe})$ 的导带为 $-0.7 \mathrm{eV}$ (图 $4 \mathrm{~d}$ ). 根据半导体带宽，我 们可以计算出 $\mathrm{NH}_{2}-\mathrm{MIL}-88 \mathrm{~B}(\mathrm{Fe})$ 的价带为 $1.2 \mathrm{eV}$. $\mathrm{NH}_{2}-\mathrm{MIL}-88 \mathrm{~B}(\mathrm{Fe})$ 在负载 GQD 前后的吸光范围变化不 大, 但负载 GQD 后, 光电流有了明显提升(图 4e), 说明 GQD 的引入提升了催化剂的光生电子-空穴对分离效 率. 鉴于 $\mathrm{GQD} / \mathrm{NH}_{2}-\mathrm{MIL}-88 \mathrm{~B}(\mathrm{Fe})$ 的种种优点, 将合成的 $\mathrm{GQD} / \mathrm{NH}_{2}-\mathrm{MIL}-88 \mathrm{~B}(\mathrm{Fe})$ 用于可见光催化二氧化碳还原. 取 $10 \mathrm{mg} \mathrm{GQD} / \mathrm{NH}_{2}-\mathrm{MIL}-88 \mathrm{~B}(\mathrm{Fe})$ 催化剂均匀分散在石 英滤膜上, 并置于固气光催化反应装置中, 加入 $2 \mathrm{~mL}$ 乙腈和三乙醇胺的混合液 (乙腈与三乙醇胺体积比为 $4: 1)$. 在 3 次抽真空充 $\mathrm{CO}_{2}$ 后, 反应体系在黑暗环境中 平衡 $1 \mathrm{~h}$. 随后, 在可见光照射下(带 $420 \mathrm{~nm}$ 截止滤光片 的 $300 \mathrm{~W} \mathrm{Xe}$ 灯), 每隔 $2 \mathrm{~h}$ 用气相色谱测一次. 如图 $4 \mathrm{f}$ 所示, GQD/ $\mathrm{NH}_{2}-\mathrm{MIL}-88 \mathrm{~B}(\mathrm{Fe})$ 在可见光照射下, 活性稳 步上升, 并在 $10 \mathrm{~h}$ 后其活性高达 $590 \mu \mathrm{mol} / \mathrm{g}$. 相对无 GQD 负载的 $\mathrm{NH}_{2}-\mathrm{MIL}-88 \mathrm{~B}(\mathrm{Fe}), \mathrm{GQD} / \mathrm{NH}_{2}-\mathrm{MIL}-$ $88 \mathrm{~B}(\mathrm{Fe})$ 的活性提升了将近 4 倍. 同时，对比实验表明， 没有催化剂的时候, 无 $\mathrm{CO}$ 产生. 另外, 当没有 $\mathrm{CO}_{2}$ 或者 光照时, 也无催化活性. 结合前述光电流数据, 我们推 

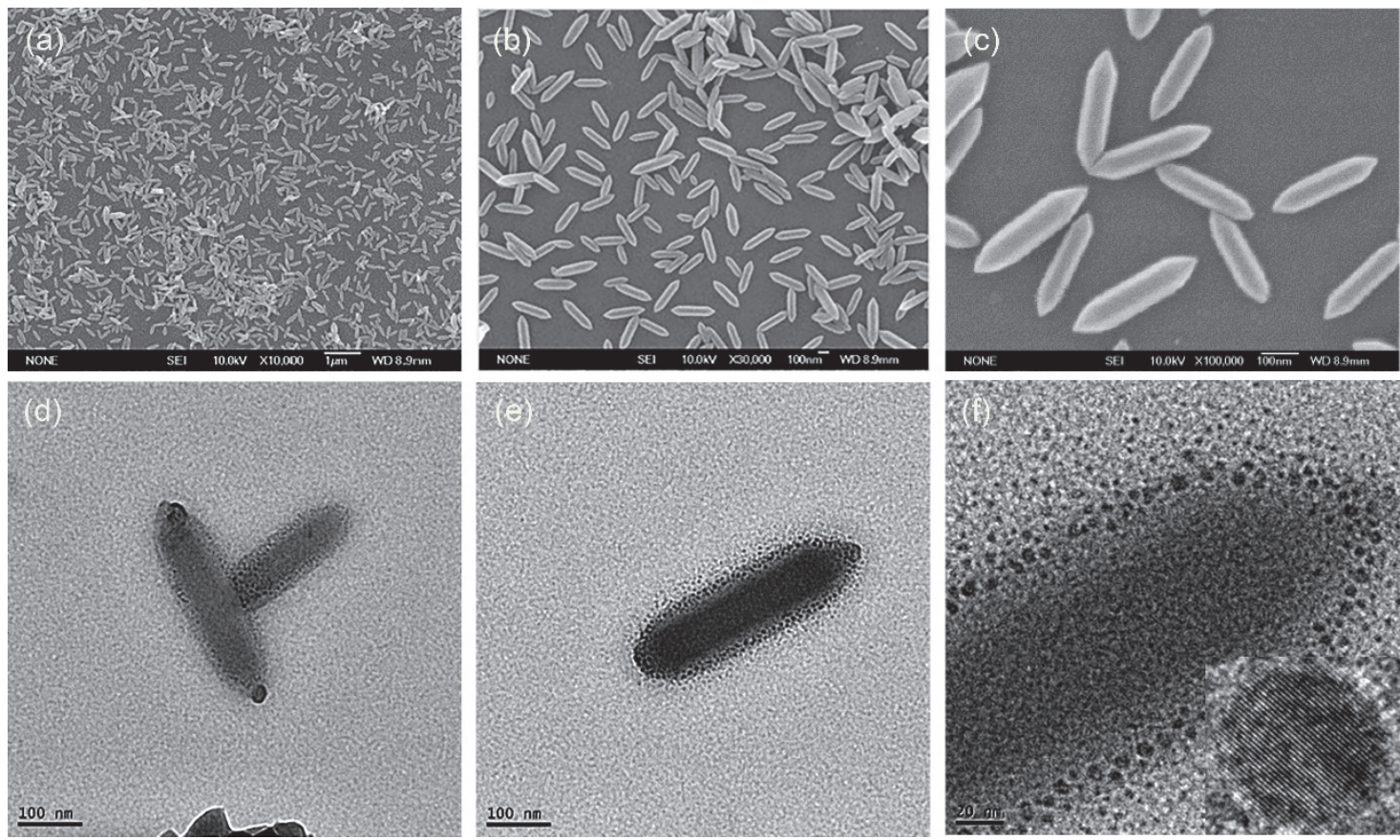

图 $3(\mathrm{a} \sim \mathrm{c}) \mathrm{NH}_{2}-\mathrm{MIL}-88 \mathrm{~B}(\mathrm{Fe})$ 的场发射扫描电镜图(标尺分别为 $1 \mu \mathrm{m}, 100 \mathrm{~nm}, 100 \mathrm{~nm}$ ); (d f f GQD/ $\mathrm{NH}_{2}-\mathrm{MIL}-88 \mathrm{~B}(\mathrm{Fe}$ )的透射电镜图(标尺分别为 $100 \mathrm{~nm}, 100 \mathrm{~nm}, 20 \mathrm{~nm}$ ), (f)图中插图为负载的 GQD 的高分辨 TEM 数据

Figure $3(\mathrm{a} \sim \mathrm{c})$ The SEM images of $\mathrm{NH}_{2}-\mathrm{MIL}-88 \mathrm{~B}(\mathrm{Fe})$ with scale bar of $1 \mu \mathrm{m}, 100 \mathrm{~nm}, 100 \mathrm{~nm}$, respectively; (d $\left.\sim \mathrm{f}\right)$ the TEM images of $\mathrm{GQD} / \mathrm{NH}_{2}-\mathrm{MIL}-88 \mathrm{~B}(\mathrm{Fe})$ with scale bar of $100 \mathrm{~nm}, 100 \mathrm{~nm}, 20 \mathrm{~nm}$, respectively. The inset in (f) is the HRTEM of GQD on NH $\mathrm{H}_{2}-\mathrm{MIL}-88 \mathrm{~B}(\mathrm{Fe})$
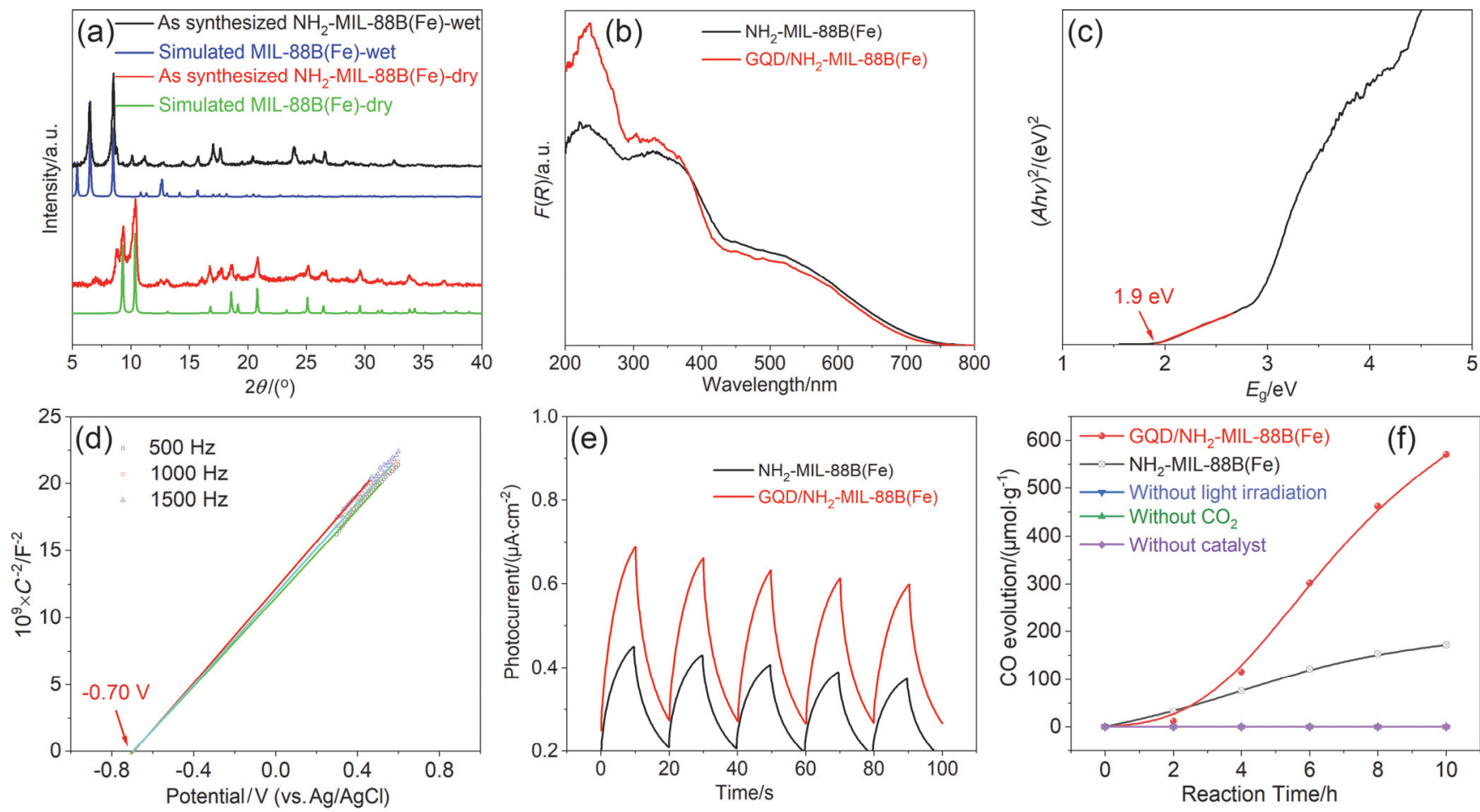

图 4 (a) $\mathrm{NH}_{2}-\mathrm{MIL}-88 \mathrm{~B}(\mathrm{Fe})$ 的粉末 X 射线衍射图; (b) GQD/ $\mathrm{NH}_{2}-\mathrm{MIL}-88 \mathrm{~B}(\mathrm{Fe})$ 和 $\mathrm{NH}_{2}-\mathrm{MIL}-88 \mathrm{~B}(\mathrm{Fe})$ 的固体紫外可见漫反射图; (c) $\mathrm{NH} \mathrm{H}_{2}-\mathrm{MIL}-88 \mathrm{~B}(\mathrm{Fe})$ 的 Tauc 线; (d) $\mathrm{NH}_{2}-\mathrm{MIL}-88 \mathrm{~B}(\mathrm{Fe})$ 的穆特-肖特基线; (e) GQD/ $\mathrm{NH}_{2}-\mathrm{MIL}-88 \mathrm{~B}(\mathrm{Fe})$ 和 $\mathrm{NH}_{2}-\mathrm{MIL}-88 \mathrm{~B}(\mathrm{Fe}$ )的光电流; (f)催化剂 GQD/NH $2-\mathrm{MIL}-88 \mathrm{~B}(\mathrm{Fe}$ ) 和 $\mathrm{NH}_{2}$-MIL-88B(Fe)对 $\mathrm{CO}_{2}$ 光催化还原效果图

Figure 4 (a) The PXRD of $\mathrm{NH}_{2}$-MIL-88B(Fe); (b) the solid UV-Vis DRS of GQD/NH $\mathrm{NH}_{2}-\mathrm{MIL}-88 \mathrm{~B}\left(\mathrm{Fe}\right.$ ) and $\mathrm{NH}_{2}-\mathrm{MIL}-88 \mathrm{~B}(\mathrm{Fe}$ ); (c) the Tauc plot of $\mathrm{NH}_{2}-\mathrm{MIL}-88 \mathrm{~B}(\mathrm{Fe})$; (d) the Mott-Schottky plots of $\mathrm{NH}_{2}-\mathrm{MIL}-88 \mathrm{~B}(\mathrm{Fe})$; (e) the photocurrents of GQD/NH $2-\mathrm{MIL}-88 \mathrm{~B}(\mathrm{Fe})$ and $\mathrm{NH} \mathrm{H}_{2}-\mathrm{MIL}-88 \mathrm{~B}(\mathrm{Fe})$; (f) photocatalytic performance of GQD/NH $-\mathrm{MIL}-88 \mathrm{~B}(\mathrm{Fe})$ and $\mathrm{NH}_{2}-\mathrm{MIL}-88 \mathrm{~B}(\mathrm{Fe})$ 
测, GQD 的引入可以极大地提升 $\mathrm{NH}_{2}-\mathrm{MIL}-88 \mathrm{~B}(\mathrm{Fe})$ 的光 生载流子分离, 进而提升了可见光光催化二氧化碳还原 活性. 光催化前后 $\mathrm{GQD} / \mathrm{NH}_{2}-\mathrm{MIL}-88 \mathrm{~B}(\mathrm{Fe})$ 的粉末 $\mathrm{X}$ 射 线衍射(PXRD)表明催化剂在催化后仍然保留原来的结 构(图 S2, 支持信息).

\section{3 结论}

通过一种简单的静电自组装法合成了一种新型石 墨烯量子点与金属有机框架复合材料, 并应用于可见光 催化二氧化碳还原. 纳米级金属有机骨架使得 GQD 高 度分散在其表面，有利于底物的接触和光吸收. 同时, 金属有机骨架表面的胺基和 GQD 上的羧基具有非常强 的静电作用力, 使得其非常稳定, 有利于电子传递, 也 有利于提升光生载流子分离能力. 合成的催化剂 GQD/ $\mathrm{NH}_{2}-\mathrm{MIL}-88 \mathrm{~B}(\mathrm{Fe})$ 较纯的 $\mathrm{NH}_{2}-\mathrm{MIL}-88 \mathrm{~B}(\mathrm{Fe})$, 在催化二 氧化碳还原制一氧化碳方面活性提升了四倍. 通过光电 流等表征, 我们推测 GQD 的引入可以极大地提升 $\mathrm{NH}_{2}-\mathrm{MIL}-88 \mathrm{~B}(\mathrm{Fe})$ 的光生载流子分离效率, 进而提升可 见光光催化二氧化碳还原活性. 该复合催化剂可以结合 金属有机骨架和石墨烯量子点的优点, 克服纯金属有机 骨架材料催化活性低的缺点. 鉴于胺基金属有机骨架材 料众多, 本方法为制备高活性金属有机骨架基材料提供 了一种参考.

\section{4 实验部分}

实验试剂和表征仪器见支持信息.

\section{1 催化剂 GQD/NH $2-\mathrm{MIL}-88 \mathrm{~B}(\mathrm{Fe})$ 的制备}

将 $\mathrm{NH}_{2}-\mathrm{MIL}-88 \mathrm{~B}(\mathrm{Fe})(50 \mathrm{mg})$ 和 $0.5 \mathrm{mg} / \mathrm{mL}(2 \mathrm{~mL})$ 的 GQD 水溶液混合, 搅拌过夜, 离心, 并用乙醇洗涤三 遍, 烘干, 得 $\mathrm{GQD} / \mathrm{NH}_{2}-\mathrm{MIL}-88 \mathrm{~B}(\mathrm{Fe})$, 量子点的质量分 数为 $2 \%$.

\section{2 光电流测试}

取 $5 \mathrm{mg}$ 样品分散在 $0.5 \mathrm{~mL}$ 无水乙醇中, 并随后加

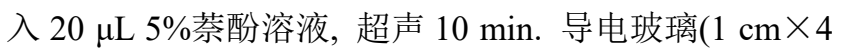
$\mathrm{cm}$ )用无水乙醇超声 $10 \mathrm{~min}$, 晾干, 并用万能表测试确 认导电面. 在导电玻璃的两端各留出 $1 \mathrm{~cm} \times 1 \mathrm{~cm}$ 的面 积, 中间部分用指甲油涂抹. 在导电玻璃的一端分 5 次 滴加样品, 每次 $10 \mu \mathrm{L}$. 将样品端置于 $0.5 \mathrm{~mol} / \mathrm{L} \mathrm{Na}_{2} \mathrm{SO}_{4}$ 水溶液中, 并用三电极法测试可见光照射下(带 $420 \mathrm{~nm}$ 截止滤光片的 $300 \mathrm{~W} \mathrm{Xe}$ 灯) 和无光照条件下的光电流强 度变化.

\section{3 光催化实验}

取 $10 \mathrm{mg}$ GQD/ $\mathrm{NH}_{2}$-MIL-88B(Fe)催化剂均匀分散 在石英过滤膜上, 置于固气光催化反应装置中, 加入 2 $\mathrm{mL}$ 乙腈和三乙醇胺的混合液(乙腈与三乙醇胺体积比 为 $4: 1$ ). 通过 3 次抽真空充 $\mathrm{CO}_{2}$, 充分去除反应装置中 的残留空气, 并在黑暗环境中平衡 $1 \mathrm{~h}$. 随后, 在可见光
照射下(带 $420 \mathrm{~nm}$ 截止滤光片的 $300 \mathrm{~W}$ Xe 灯), 每隔 $2 \mathrm{~h}$ 用气相色谱测一次. $\mathrm{NH}_{2}-\mathrm{MIL}-88 \mathrm{~B}(\mathrm{Fe})$ 的测试方法同上.

\section{References}

[1] Meng, D. L.; Zhang, M. D.; Si, D. H.; Mao, M. J.; Hou, Y.; Huang, Y. B.; Cao, R. Angew. Chem., Int. Ed. 2021, 60, 25485.

[2] Yi, J. D.; Si, D. H.; Xie, R.; Yin, Q.; Zhang, M. D.; Wu, Q.; Chai, G. L.; Huang, Y. B.; Cao, R. Angew. Chem., Int. Ed. 2021, 60, 17108.

[3] Yi, J. D.; Xie, R.; Xie, Z. L.; Chai, G. L.; Liu, T. F.; Chen, R. P.; Huang, Y. B.; Cao, R. Angew. Chem., Int. Ed. 2020, 59, 23641.

[4] Zou, Y. H.; Huang, Y. B.; Si, D. H.; Yin, Q.; Wu, Q. J.; Weng, Z.; Cao, R. Angew. Chem., Int. Ed. 2021, 60, 20915.

[5] Diercks, C. S.; Liu, Y.; Cordova, K. E.; Yaghi, O. M. Nat. Mater 2018, 17, 301 .

[6] Zhang, W.; Mohamed, A. R.; Ong, W. J. Angew. Chem., Int. Ed. 2020, 59, 22894.

[7] Wang, X. S.; Li, L.; Li, D.; Ye, J. Solar RRL 2020, 4, 1900547.

[8] Hu, J.; Chen, D.; Mo, Z.; Li, N.; Xu, Q.; Li, H.; He, J.; Xu, H.; Lu, J. Angew. Chem., Int. Ed. 2019, 58, 2073.

[9] Yu, H.; Li, J.; Zhang, Y.; Yang, S.; Han, K.; Dong, F.; Ma, T.; Huang, H. Angew. Chem., Int. Ed. 2019, 58,3880 .

[10] Hong, D.; Kawanishi, T.; Tsukakoshi, Y.; Kotani, H.; Ishizuka, T.; Kojima, T. J. Am. Chem. Soc. 2019, 141, 20309.

[11] Yu, H.; Haviv, E.; Neumann, R. Angew. Chem., Int. Ed. 2020, 59, 6219.

[12] Liang, J.; Wu, Q.; Huang, Y. B.; Cao, R. EnergyChem 2021, 3, 100064.

[13] Wang, X.-S.; Liang, J.; Li, L.; Lin, Z.-J.; Bag, P. P.; Gao, S.-Y.; Huang, Y.-B.; Cao, R. Inorg. Chem. 2016, 55, 2641.

[14] Wang, X.-S.; Chen, C.-H.; Ichihara, F.; Oshikiri, M.; Liang, J.; Li, L.; Li, Y.; Song, H.; Wang, S.; Zhang, T.; Huang, Y.-B.; Cao, R.; Ye, J. Appl. Catal. B: Environ. 2019, 253, 323.

[15] He, C.; Liang, J.; Zou, Y.-H.; Yi, J.-D.; Huang, Y.-B.; Cao, R. Natl. Sci. Rev. 2021, DOI:10.1093/nsr/nwab157.

[16] Xiong, W.; Li, H.; You, H.; Cao, M.; Cao, R. Natl. Sci. Rev. 2020, 7 , 609.

[17] Li, X.; Yan, B.; Huang, W.; Fu, L.; Sun, X.; Lv, A. Acta Chim. Sinica 2021, 79, 459 (in Chinese). (李旭飞, 间保有, 黄维秋, 浮历沛, 孙 宪航，吕爱华，化学学报, 2021, 79, 459.)

[18] Wu, Q.; Zhang, C.; Sun, K.; Jiang, H.-L. Acta Chim. Sinica 2020 78,688 (in Chinese). (吴浅耶, 张晨䂀, 孙康, 江海龙, 化学学报, 2020, 78,688 .)

[19] Lin, Z.-J.; Cao, R. Acta Chim. Sinica 2020, 78, 1309. (林祖金, 曹 荣, 化学学报, 2020, 78, 1309)

[20] Zhang, X.; Li, X.; Xiong, W.; Li, H.; Cao, R. Acta Chim. Sinica 2021, 79, 180 (in Chinese). (张晓萌，李希雅，熊晚枫，李红芳，曹 荣, 化学学报, 2021, 79, 180.)

[21] Pan, Y.; Qian, Y.; Zheng, X.; Chu, S. Q.; Yang, Y.; Ding, C.; Wang, X.; Yu, S. H.; Jiang, H. L. Natl. Sci. Rev. 2021, 8, nwaa224.

[22] Wang, H.-N.; Zou, Y.-H.; Sun, H.-X.; Chen, Y.; Li, S.-L.; Lan, Y.-Q. Coord. Chem. Rev. 2021, 438, 213906.

[23] Huang, Q.; Liu, J.; Feng, L.; Wang, Q.; Guan, W.; Dong, L. Z.; Zhang, L.; Yan, L. K.; Lan, Y. Q.; Zhou, H. C. Natl. Sci. Rev. 2020, 7,53 .

[24] Fu, Y.; Sun, D.; Chen, Y.; Huang, R.; Ding, Z.; Fu, X.; Li, Z. Angew. Chem., Int. Ed. 2012, 51, 3364.

[25] Wang, D.; Huang, R.; Liu, W.; Sun, D.; Li, Z. ACS Catal. 2014, 4 4254.

[26] Xu, H. Q.; Hu, J.; Wang, D.; Li, Z.; Zhang, Q.; Luo, Y.; Yu, S. H.; Jiang, H. L. J. Am. Chem. Soc. 2015, 137, 13440.

[27] Zhang, H.; Wei, J.; Dong, J.; Liu, G.; Shi, L.; An, P.; Zhao, G.; Kong, J.; Wang, X.; Meng, X.; Zhang, J.; Ye, J. Angew. Chem., Int. Ed. 2016, $55,14310$.

[28] Chen, E. X.; Qiu, M.; Zhang, Y. F.; Zhu, Y. S.; Liu, L. Y.; Sun, Y. Y.; Bu, X.; Zhang, J.; Lin, Q. Adv. Mater. 2018, 30, 1704388.

[29] Dong, L. Z.; Zhang, L.; Liu, J.; Huang, Q.; Lu, M.; Ji, W. X.; Lan, Y. Q. Angew. Chem., Int. Ed. 2020, 59, 2659.

[30] Chen, Q.; Kuang, Q.; Xie, Z. Acta Chim. Sinica 2021, 79, 10 (in Chinese). (陈钱, 匡勤, 谢兆雄, 化学学报, 2021, 79, 10.)

[31] Zhao, H.; Wang, X.; Feng, J.; Chen, Y.; Yang, X.; Gao, S.; Cao, R. Catal. Sci. Technol. 2018, $8,1288$.

[32] Shi, L.; Wang, T.; Zhang, H.; Chang, K.; Ye, J. Adv. Funct. Mater. $\mathbf{2 0 1 5}, 25,5360$. 
[33] Wang, X.; Zhao, X.; Zhang, D.; Li, G.; Li, H. Appl. Catal. B: Environ. 2018, 228, 47.

[34] Han, Z.; Fu, Y.; Zhang, Y.; Zhang, X.; Meng, X.; Zhou, Z.; Su, Z. Dalton Trans. 2021, 50, 3186.

[35] Jiang, Z.; Xu, X.; Ma, Y.; Cho, H. S.; Ding, D.; Wang, C.; Wu, J.; Oleynikov, P.; Jia, M.; Cheng, J.; Zhou, Y.; Terasaki, O.; Peng, T.; Zan, L.; Deng, H. Nature 2020, 586, 549.

[36] Li, H.; Kang, Z.; Liu, Y.; Lee, S.-T. J. Mater. Chem. 2012, 22, 24230 .

[37] Lim, S. Y.; Shen, W.; Gao, Z. Chem. Soc. Rev. 2015, 44, 362.

[38] Wang, J.; Cheng, C.; Huang, Y.; Zheng, B.; Yuan, H.; Bo, L.; Zheng, M.-W.; Yang, S.-Y.; Guo, Y.; Xiao, D. J. Mater. Chem. C 2014, 2 , 5028.

[39] Aguilera-Sigalat, J.; Bradshaw, D. Coord. Chem. Rev. 2016, 307, 267.
[40] Martindale, B. C.; Hutton, G. A.; Caputo, C. A.; Reisner, E. J. Am. Chem. Soc. 2015, 137, 6018.

[41] Dong, Y.; Han, Q.; Hu, Q.; Xu, C.; Dong, C.; Peng, Y.; Ding, Y.; Lan, Y. Appl. Catal. B: Environ. 2021, 293, 120214.

[42] Ming, H.; Ma, Z.; Liu, Y.; Pan, K.; Yu, H.; Wang, F.; Kang, Z. Dalton Trans. 2012, 41, 9526.

[43] Liu, J.; Liu, Y.; Liu, N.; Han, Y.; Zhang, X.; Huang, H.; Lifshitz, Y.; Lee, S. T.; Zhong, J.; Kang, Z. Science 2015, 347, 970.

[44] Bian, J.; Huang, C.; Wang, L.; Hung, T.; Daoud, W. A.; Zhang, R. ACS Appl. Mater. Inter. 2014, 6, 4883.

[45] Li, H.; He, X.; Kang, Z.; Huang, H.; Liu, Y.; Liu, J.; Lian, S.; Tsang, C. H.; Yang, X.; Lee, S. T. Angew. Chem., Int. Ed. 2010, 49, 4430.

$(\mathrm{Lu}, \mathrm{Y}$ ) 